\title{
Bulletin des médecins suisses - nouvelle formule
}

Pour garder un secret, le mieux est de le glisser dans la rubrique des directives pour les auteurs, relève Frank Davidoff dans la dernière édition de Health Affairs [1], parce que de toutes façons, personne ne la lit. Son expérience en tant qu'ancien rédacteur de la revue Annals of Internal Medicine est certainement aussi partagée par des rédactions moins prestigieuses.

La rédaction du Bulletin des médecins suisses (BMS), avec une interprétation le plus souvent large de ses propres directives à l'intention des auteurs, a elle aussi contribué au peu d'attention portée à celle-ci. Un exemple de cette attitude libérale est fourni par les lettres de lecteurs qu'il nous arrive malgré tout de publier même si elles dépassent largement la longueur prescrite. Nous ne saurions, en effet, jouer les maîtres d'école. Cette manière de voir me semblait justifiée aussi parce qu'elle est l'expression d'une approche résolument antidogmatique de la politique professionnelle et de santé publique, la tâche première de la nouvelle rédaction étant d'offrir aux membres de la FMH la possibilité d'un échange de vues libre et équilibré. Cela dit, des raisons purement pratiques ont également et régulièrement rendu nécessaires quelques retouches sur le plan rédactionnel: nouveaux besoins d'information, changements de personnel au sein de la rédaction, restrictions budgétaires, nouveaux concepts éditoriaux, à l'image du Swiss Medical Forum. Bref, il fallait éviter les pédanteries rédactionnelles et mettre l'accent plutôt sur des principes et des modes de travail utiles à long terme: équipe rédactionnelle décentralisée, indépendance rédactionnelle par rapport à la maison d'édition et à l'éditeur, liberté d'opinion des auteurs, appréciation des manuscrits selon leur seule qualité, originalité et actualité, «peer review» des articles non médi-caux également. Ces principes ont-ils fait leurs preuves?

Un succès se doit d'être relevé: nous avons gagné un nombre grandissant d'auteurs et un lectorat en progression dans les domaines les plus divers de l'activité médicale et non médicale. Hormis le corps médical en effet, nous donnons la parole à des auteurs émanant d'autres domaines: économie, droit, politique, théologie, soins infirmiers, informatique et histoire. De même, nous traitons de nombreux thèmes sous plusieurs angles, animant ainsi la controverse et incitant aux commentaires.

Il est parfois surprenant de voir l'importance que prend la parution (ou la non-parution) d'un article dans le BMS, notamment à l'approche d'un vote populaire ou d'une décision parlementaire. Ces expériences sont en quelque sorte le signe que le BMS a un impact grandissant dans le débat en matière de politique de la santé. Et c'est bien ainsi.

Le fait le plus marquant de ces dernières années est, à mon avis, que la liberté d'expression des membres de la FMH dans le BMS soit entrée dans la norme. Du moins, le plus grand nombre des intervenants semble avoir compris que les articles politiques s'appuyant sur des faits et dénués d'attaques personnelles ne sont pas refusés, même s'ils défendent des intérêts particuliers parfaitement opposés à la doctrine «officielle». Il suffit de prendre pour exemple la récente histoire de la votation générale. Cette transparence n'a nui en aucune manière à la voix officielle de la FMH, au contraire, puisque cette expression ne prend tout son sens que dans le contexte d'un corps médical éminemment pluraliste. En tant que voix solitaire, elle ne recevrait que peu de crédibilité. Je souhaite que le BMS contribue ainsi, même modestement, au bon fonctionnement démocratique de notre organisation professionnelle.

La diversité d'opinions a aussi son revers puisqu'elle peut déraper dans l'insipide et l'indifférence. Il a donc semblé approprié, dans la foulée de la politique rédactionnelle jusqu'ici pratiquée, que l'on pourrait qualifier d'ouverture, de rénover le concept du BMS, de remanier le contexte graphique et rédactionnel et d'accorder à nouveau plus de place à certaines règles de conduite.

Cette édition inaugure la nouvelle conception. Le changement le plus spectaculaire réside dans un graphisme plus sobre, une meilleure lisibilité et une structuration plus claire des articles. Dans cet esprit, de nouvelles rubriques affinent la répartition thématique. La rubrique «Politique, économie et droit» devient «Politique» tout court, et transmet les communications officielles de la FMH. Elle est ouverte également à toutes les organisations de base de la 
FMH, ainsi qu'à d'autres organisations et particuliers, selon entente avec la rédaction. Nous avons l'intention d'aborder tous les sujets de politique professionnelle, de manière plus systématique, fiable et transparente. Ainsi, nos informations sur l'agenda politique, par exemple, paraîtront désormais le plus tôt possible pour permettre un débat ouvert et honnête sur les thèmes concernés et les votes à venir. Les articles sur le droit et l'économie sont nouvellement rassemblés sous une deuxième rubrique importante intitulée «Sciences» qui remplace la précédente rubrique «Médecine» (devenant caduque en raison de la suppression des articles sur la formation continue médicale paraissant désormais dans Swiss Medical Forum). La rubrique «Sciences» est consacrée aux disciplines qui, sur le plan de la politique professionnelle, peuvent être considérées comme disciplines annexes. Concrètement, elle a pour thèmes des travaux originaux, des articles sur la formation continue, des commentaires et des débats sur les thèmes suivants: assurance qualité, recommandations pour la pratique clinique («guidelines»), éducation médicale, exercice de la profession médicale (Medical profession and workforce), santé publique, économie, droit, éthique, histoire, informatique médicale. C'est une liste d'axes prioritaires sans prétention d'exhaustivité. L'évaluation des manuscrits se fonde sur des critères d'ordre scientifique. Les recommandations pour la pratique clinique doivent en outre, sur le plan de la teneur et de la forme, répondre aux critères de la directive FMH en la matière [2]. Tous les articles de cette rubrique sont revus par au moins un expert externe («peer review»). Nous attachons une valeur particulière, comme il se doit pour toute publication sérieuse, à la transparence des associations d'intérêts. La troisième partie du bulletin passe du titre de «Distillerie» à celui de «Points de vue». Cette rubrique est destinée à la publication d'interviews, d'essais et de prises de position individuelles, parvenus spontanément ou à notre demande, dans tous les domaines du monde médical et de la santé. Une place plus grande sera accordée au «Courrier des lecteurs». Placé au début, sur deux colonnes, à la suite de l'éditorial, il témoigne de la valeur que nous accordons au lectorat et à son opinion. Cette revalorisation graphique demande néanmoins que l'on s'en tienne strictement à la longueur maximale des textes pour garder un minimum de contrôle sur la taille du bulletin. D'autres articles, l'éditorial en particulier, seront en principe rédigés par des invités de la rédaction ou, en accord avec celle-ci, de manière plus spontanée. Tel est l'aperçu du nouveau concept et des points essentiels des nouvelles directives pour les auteurs. Pour en savoir plus, veuillez consulter la page 810 .

Ces changements d'ordre conceptuels ne seraient que brouillons sans le personnel nécessaire à les réaliser. Nous sommes heureux de pouvoir renforcer notre équipe rédactionnelle; d'autres détails à cet égard sont fournis dans le rapport annuel de la FMH, page 776 de la présente édition du BMS. Il est réjouissant, également, de pouvoir compter sur des experts externes dans le domaine rédactionnel. Il nous a même été possible de réunir une petite équipe de collaborateurs réguliers pour la rubrique «éthique» (cf. Impressum).

Dernière nouveauté de taille: pour la dernière fois, vous trouvez en annexe de cette édition le calendrier des congrès sous sa forme imprimée. Une liste sur papier ne saurait désormais concurrencer les possibilités quasi illimitées d'une banque électronique de données. A l'adresse www.medkong.ch, les mêmes renseignements vous sont livrés sans devoir vous perdre dans une masse d'informations, mais en cherchant de manière ciblée ce qui répond à vos aspirations et à vos intérêts. Un simple formulaire permet aux organisateurs de sessions de formation continue d'enregistrer les manifestations auxquelles vous participez. Une autre innovation importante réside dans l'indication des crédits par discipline médicale, ainsi que dans les possibilités de recherche.

Par le biais de cette restructuration du BMS, nous avons engagé toute une série de modifications que nous espérons utiles. Tout en restant ouverts, comme par le passé, à la critique et aux suggestions, nous continuerons d'apporter toutes les améliorations nécessaires.

Dr Markus Trutmann rédacteur en chef du Bulletin des médecins suisses

1 Davidoff F. Between the lines: navigating the uncharted territory of industry-sponsored research. Health Affairs 2002;21(2):235-42. http://www.healthaffairs.org/freecontent/s29.htm

2 Directive pour les guides de pratique médicale. Bull Méd Suisses 1999;80(14):831-3 (renommée par la suite «Directive concernant les recommandations pour la pratique clinique»). 